\title{
Endogenous Cushing's Syndrome in a Patient with Systemic Lupus Erythematosus
}

\author{
EunJin Kang ${ }^{1}$, Su-Jin Moon², Kyung ho Moon ${ }^{1}$, Deok-jae Han ${ }^{1}$, Jain Lee ${ }^{1}$, Sang Mi Ro ${ }^{1}$, Jang-Won Son ${ }^{1}$, Sung-Rae Kim¹, Jun-Ki Min², \\ Soon Jib Yoo ${ }^{1, *}$ \\ Departments of ${ }^{1}$ Endocrinology and Metabolism, ${ }^{2}$ Rheumatology, College of Medicine, Bucheon St. Mary's Hospital, The Catholic University of Korea, Bucheon, \\ Korea
}

Systemic lupus erythematosus is an autoimmune disease for which glucocorticoids are the mainstay of treatment. Cushing's syndrome is caused by glucocorticoid excess, which can be either exogenous or endogenous. Although iatrogenic Cushing's syndrome is the most common form, especially in patients undergoing glucocorticoid treatment, endogenous glucocorticoid excess should be considered because it has a different treatment strategy. We describe a 51-year old woman with a longstanding history of SLE. She was treated with steroid and cytoxan pulse therapy and plasmapheresis. Her lupus activity had been stable for 7 years with low-dose glucocorticoid treatment. She showed excessive weight gain, easy bruising, moon facies, truncal obesity, acne, and menstrual disorder. Given her history of long-term steroid therapy, iatrogenic Cushing's syndrome was considered the most likely diagnosis; however, worsening features of Cushing's syndrome with a minimal dose of glucocorticoid led us to diagnose endogenous Cushing's syndrome due to a left adrenal adenoma. The patient underwent laparoscopic left adrenalectomy. Her SLE was controlled with transient low-dose glucocorticoid treatment, and her lupus activity remained stable without glucocorticoid treatment. This is the first reported case of concomitant endogenous Cushing's syndrome in a patient with preexisting SLE in Korea. This case shows the importance of differential diagnosis including exogenous Cushing's syndrome and endogenous Cushing's syndrome in autoimmune disease patients with glucocorticoid therapy.

Key words: Cushing's syndrome, Systemic lupus erythematosus, Glucocorticoid therapy, Differential diagnosis

\section{Introduction}

Cushing's syndrome resulting from exogenous glucocorticoids is common. ${ }^{1}$ However, endogenous glucocorticoid excess in a patient receiving steroids for a preexisting autoimmune disease could be misinterpreted as iatrogenic. ${ }^{2}$ For the most part, exogenous Cushing's syndrome presents with the same signs and symptoms as endogenous Cushing's syndrome. There are, nevertheless, a few important differences in presentation. ${ }^{1}$

Systemic lupus erythematosus (SLE) is an autoimmune disease for which glucocorticoids, which have both immunosuppressive and anti-inflammatory effects, are an effective therapy. We report an interesting first case of a patient with SLE accompanied by Cushing's syndrome due to adrenal adenoma in Korea.

\section{Case Report}

A 51-year old Korean woman with a longstanding history of SLE from the age of 27 years initially presented in 1991 with malar rash, polyarthralgia, myalgia, proteinuria, positive antinuclear antibodies (speckled type), anti-DNA antibodies, rheumatoid factor, and LE cells. From these findings, she was diagnosed with SLE. She was treated with a glucocorticosteroid (prednisolone). She underwent steroid pulse therapy in 1992 and plasmapheresis and cytoxan pulse therapy in 1993. Her lupus activity remained stable for 7 years before she presented to our hospital in February 2010, at which point she was being treated with $2.5 \mathrm{mg}$ of prednisolone per day. She was referred with a clinical impression of Cushing's syndrome because of weight gain (10 kg/year), easy bruising, development of moon facies,
Corresponding author Soon Jib Yoo (iD http://orcid.org/0000-0002-9932-4130 Department of Endocrinology, College of Medicine, Bucheon St. Mary's Hospital, The Catholic University of Korea, 327 Sosa-ro, Wonmi-gu, Bucheon 14647, Korea Tel +82-32-340-7011 Fax+82-32-340-2669 E-mail sjyoo@catholic.ac.kr Received Oct. 14, 2015 Reviewed Oct. 22, 2015 Accepted Oct. 25, 2015
Copyright (C) 2016 Korean Society for the Study of Obesity

() This is an Open Access article distributed under the terms of the Creative Commons Attribution Non-Commercial License (http://creativecommons.org/licenses/by-nc/4.0/) which permits unrestricted non-commercial use, distribution, and reproduction in any medium, provided the original work is properly cited. 

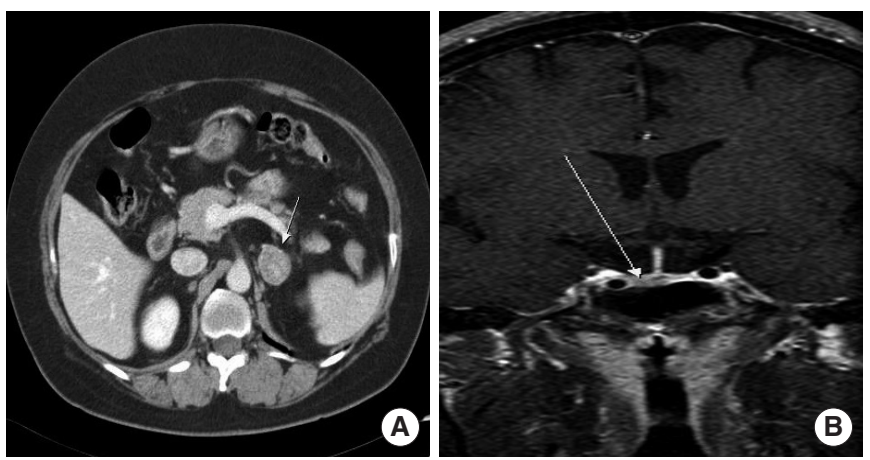

Fig. 1. Abdominal computed tomography (A) suggested a 3-cm sized left adrenal adenoma (arrow). Magnetic resonance imaging of the sella (enhanced coronal T1$\mathrm{WI}$ ) showed a $5 \times 7 \times 5 \mathrm{~mm}(\mathrm{AP} \times \mathrm{T} \times$ height) pituitary microadenoma $(\mathrm{B})$ in the right posterior aspect of the pituitary fossa.

truncal obesity, acne, and menstrual disorder. She was admitted to our hospital for Cushing's syndrome evaluation because of positive findings on a screening test (increased 24-hour urine free cortisol; 1,494.74 $\mu \mathrm{g} /$ day) and a 3-cm-sized left adrenal mass (Fig. 1A) detected in our outpatient department.

On admission, her blood pressure was 110/70 mm Hg. Height was $156.3 \mathrm{~cm}$, and body weight was $82.35 \mathrm{~kg}$. Her body mass index was $33.71 \mathrm{~kg} / \mathrm{m}^{2}$. Complete blood cell count showed the following: white cell count $12,090 \times 10^{9} / \mathrm{L}$ with $84 \%$ neutrophils, hemoglobin $14.6 \mathrm{~g} /$ $\mathrm{dl}$, and platelet count $227 \times 10^{9} / \mathrm{L}$. Evaluation of serum chemistry revealed lactate dehydrogenase $627 \mathrm{IU} / \mathrm{L}, \mathrm{C}$-reactive protein $0.3 \mathrm{mg} / \mathrm{L}$, and an erythrocyte sedimentation rate of $5 \mathrm{~mm} / \mathrm{h}$. Anti-nuclear antibody was 1:80 and 1:160 for homogenous and anti-cytoplasmic types, respectively. Assays for anti-DNA (anti-deoxyribonucleic acid), antiCCP (anti-citrullinated protein), anti-RNP (anti-ribonucleoprotein), anti-Sm (anti-Smith), anti-SS-A/B (anti-Sjogren's-syndrome-related antigen $\mathrm{A} / \mathrm{B}$ ), anti-Ro-52 (TRIM21, tripartite motif-containing protein 21), anti-Scl-70 (anti-topoisomerase I), anti-Jo-1 (anti-histidyl-t ribonucleic acid synthetase), anti-centromere B, anti-nucleosomes, anti-histones, lupus anticoagulant, anti-cardiolipin, anti-platelet antibodies, and rheumatoid factor were all negative. Concentrations of serum C3 and C4 were normal. Plasma ACTH level at 8:00 am and $11: 00 \mathrm{pm}$ was 13.52 and $16.58 \mathrm{pg} / \mathrm{mL}$, respectively, and cortisol level at 8:00 am and 11:00 pm was 21.52 and $21.02 \mu \mathrm{g} / \mathrm{dL}$, respectively; both were consistently high without circadian changes. The twentyfour hour urinary excretion level of 17-hydroxycorticosteroids (17OHCS) was $10.7 \mathrm{mg} /$ day. Serum cortisol was not suppressed by lowdose ( $2 \mathrm{mg}$ for 2 days) or high-dose ( $8 \mathrm{mg}$ for 2 days) dexamethasone
Table 1. Hormonal evaluations

\begin{tabular}{llrcc}
\hline & & $\begin{array}{c}\text { ACTH } \\
(\mathrm{pg} / \mathrm{mL})\end{array}$ & $\begin{array}{c}\text { Cortisol } \\
(\mu \mathrm{g} / \mathrm{dL})\end{array}$ & $\begin{array}{c}24 \mathrm{hr} \text { UFC/17-OHCS/17-KS } \\
(\mu \mathrm{g} / \text { day, } \mathrm{mg} / \text { day, } \mathrm{mg} / \text { day })\end{array}$ \\
\hline Baseline study & $8: 00 \mathrm{AM}$ & 13.52 & 21.52 & \\
& $23: 00 \mathrm{PM}$ & 16.58 & 21.02 & \\
+Dexamethasone & $0.5 \mathrm{mg} \mathrm{q} 6 \mathrm{hr}$ for & 6.44 & 22.95 & $1287 / 10.9 / 4.7$ \\
& 2 days & & & \\
& 2 mg q 6 hr for & 9.56 & 21.44 & $854 / 11.6 / 6.2$ \\
& 2 days & & & \\
\hline
\end{tabular}

administration (Table 1). Levels of other hormones, including 24 hours urine metanephrine, normetanephrine, vanillylmandelic acid (VMA), epinephrine, and norepinephrine, were within normal ranges. Magnetic resonance imaging of the sella was performed based on the differential diagnosis and showed a pituitary microadenoma in the right posterior aspect of the pituitary fossa (Fig. 1B). A basal pituitary hormonal study showed normal findings. From these findings, the patient was diagnosed with Cushing's syndrome caused by a left adrenal adenoma.

The patient underwent laparoscopic left adrenalectomy in April 2010. A tan-yellow $3 \times 3 \mathrm{~cm}$ solid mass was found in the left adrenal cortex. Microscopic examination revealed that the mass was an adrenal cortical adenoma. After surgery, she received $15 \mathrm{mg} /$ day of prednisolone as replacement therapy for 1 month and successfully tapered after 3 years. She did not experience SLE exacerbation. Five years after surgery, she remained healthy with relief of her symptoms. Her last abdominal computed tomography in 2012 showed no evidence of recurrence of the adrenal lesion. The last follow-up sella MRI in March 2015 showed regression of the pituitary adenoma.

\section{Discussion}

Endogenously hypersecreted cortisol in autoimmune disease is unlikely to develop in Cushing's syndrome. In addition, glucocorticoids are a mainstay of treatment in autoimmune disease. It is difficult for clinicians to distinguish endogenous Cushing's syndrome from iatrogenic Cushing's caused by exogenously administered glucocorticoids. Therefore, it is important to evaluate endogenous glucocorticoid secretion in patients who have been treated with glucocorticoids if Cushing's signs, including hypertension, become rapidly overt or the activity of autoimmune disease is unexpectedly silent. The symptoms related to concomitant endogenous Cushing's syndrome are aggravated hypertension, hypokalemia, and pathologic 
fractures, with or without specific features such as central obesity and moon facies. These conditions are clues to endogenous glucocorticoid excess in patients not undergoing steroid therapy. Such findings could also lead to suspicion of iatrogenic Cushing's syndrome in a patient receiving steroid therapy for autoimmune disease; however, some clinical manifestations show different prevalences in iatrogenic vs. endogenous Cushing's syndrome. Hypertension, hirsutism, and oligomenorrhea/amenorrhea are more prevalent in endogenous Cushing's syndrome, whereas features such as glaucoma, avascular necrosis, and pancreatitis are more common in iatrogenic Cushing's syndrome. The clinical manifestations of iatrogenic Cushing's syndrome are more striking than those of endogenous Cushing's, which occurs gradually. In our case, the patient showed gradual development of aggravated hypertension and menstrual irregularities over 1 year. Based on the possibility of endogenous cortisol excess, we conducted an evaluation and differential diagnosis of Cushing's syndrome.

Concurrent endogenous Cushing's syndrome and SLE are extremely rare, and only 4 cases have been reported in the literature, including the present case. ${ }^{2,45}$ Notably, three of these patients were Japanese females; to the best of our knowledge, our patient was the first reported in Korea. The previously reported patients were diagnosed as having Cushing's syndrome due to unilateral adrenal adenoma in two patients and bilateral adenomas in one. When Cushing's syndrome was finally diagnosed, all of the patients were in a remissive state of SLE. One patient showed apparent remission when Cushing's syndrome was diagnosed. It was believed that the remission was due to endogenously secreted cortisol. After treatment of Cushing's syndrome with adrenalectomy, the patient experienced exacerbation of SLE and required intensive steroid therapy. A few cases of rheumatoid arthritis patients with Cushing's syndrome also showed a similar course, with remission after diagnosis of Cushing's syndrome and relapse after treatment of hypercortisolism. ${ }^{6,7}$ Two other patients showed favorable control of the preexisting autoimmune disease after treatment of concomitant Cushing's syndrome. (One patient underwent a second operation for a remnant adrenal tumor on the other side due to relapse of Cushing's syndrome.)

It is speculated that an endogenous excess of glucocorticoids could suppress autoimmune activities. However, the varying courses of preexisting autoimmune disease activity have to be considered. A rebound in abnormal autoimmune activity may be induced by abrupt reduction in endogenous glucocorticoids after treatment for Cushing's syndrome. Careful attention must be paid to the development of occult autoimmune disease after treatment for Cushing's disease, since excess glucocorticoids can suppress autoimmune processes. Shimizu et al. ${ }^{2}$ assessed elevated endogenous glucocorticoids and replaced them with a sufficient dosage of prednisolone, using the 24hour urinary excretion level of 17-OHCS. ${ }^{2}$ The present patient's status is closely monitored with 24-hour urinary free cortisol and the 1-mg dexamethasone suppression test. After adrenalectomy, the patient has not shown any evidence of recurrence of Cushing's syndrome for 5 years. Over 1 year, glucocorticoid replacement was completely tapered, and she has remained in a remissive state of SLE without flares.

This is the first reported case of concomitant endogenous Cushing's syndrome with preexisting SLE in Korea. However, there is a possibility of overlooking Cushing's syndrome cases and iatrogenic Cushing's syndrome in autoimmune disease patients with glucocorticoid therapy. Careful consideration is needed for the differential diagnosis of exogenous Cushing's syndrome and endogenous Cushing's syndrome in such patients.

\section{Conflicts of Interest}

There is no conflict of interest that could be perceived as prejudicing the impartiality of the research reported.

\section{References}

1. Hopkins RL, Leinung MC. Exogenous Cushing's syndrome and glucocorticoid withdrawal. Endocrinol Metab Clin North Am 2005;34:371-84, ix.

2. Shimizu M, Kawata M, Okada T, Yuu H, Kurahashi T, Yamanaka K, et al. Concomitant Cushing's syndrome due to adrenal adenoma in a patient with systemic lupus erythematosus. Intern Med 2002;41:1044-6.

3. Ghrousos GP. Clucocorticoid therapy. In: Felig P, Frohman LA, editors. Endocrinology and Metabolism. 4th ed. New York: McGraw-Hill; 2001. p. 609-32.

4. Arima K, Higuchi M, Yoshizawa S, Horiuchi T, Nagasawa K, Nakashima $\mathrm{H}$, et al. Improvement of systemic lupus erythematosus activity by the association of delayed onset Cushing's syndrome. J 
Rheumatol 1998;25:2456-8.

5. Katayama A, Otsuka F, Tanabe K, Tsukamoto N, Yamanaka R, Matsumoto Y, et al. Unexpected occurrence of adrenal Cushing's syndrome in a patient with systemic lupus erythematosus. Hypertens Res 2011;34:662-3.

6. Senécal JL, Uthman I, Beauregard H. Cushing's disease-induced remission of severe rheumatoid arthritis. Arthritis Rheum 1994;37: 1826.

7. Yakushiji F, Kita M, Hiroi N, Ueshiba H, Monma I, Miyachi Y. Exacerbation of rheumatoid arthritis after removal of adrenal adenoma in Cushing's syndrome. Endocr J 1995;42:219-23. 